\title{
Clinical Study of Facial Wrinkle Treatment with Fractional Microneedle Radio Frequency System
}

\author{
Hyoun Jun Park ${ }^{1}$ \\ Hyoung Moon Kim² \\ Myung Jin $\mathrm{Oh}^{3}$
}

${ }^{1}$ Maylin Clinic, Seoul, Korea

${ }^{2}$ Miaero Medical Aesthetic Group, Seoul, Korea

${ }^{3}$ Dr. Oh S. Skin LASER Clinic, Seoul, Korea
Received November 20, 2014

Revised December 12, 2014

Accepted December 20, 2014

\section{Correspondence}

Hyoun Jun Park

Maylin Clinic, 450 Apgujeong-dong, Gangnam-gu, Seoul 135-901, Korea

Tel: +82-2-563-0000

Fax: +82-2-542-7201

E-mail: parmanidanaver.com

(C) Korean Society for Laser Medicine and Surgery

(c) This is an open access article distributed under the terms of the Creative Commons Attribution NonCommercial License (http://creativecommons.org/ licenses/by-nc/3.0) which permits unrestricted noncommercial use, distribution, and reproduction in any medium, provided the original work is properly cited.

\author{
Background and Objectives \\ Noninvasive radio frequency (RF) devices have been used in treatment of \\ facial wrinkles. Fractional microneedle RF system was studied to assess \\ the clinical efficacy and safety in treatment of facial wrinkles.

\section{Materials and Methods} \\ A total of 204 patients $(80$ male, 124 female; mean age 47.3, range 26-68; \\ Fitzpatrick skin type III to type IV) with facial wrinkle were treated with 1-3 \\ sessions of fractional microneedle RF system at an average interval of 4 \\ weeks at three study centers in Korea.
}

\section{Results}

Of the 204 patients, 54 had grade 4 clinical improvement, 86 had grade 3 improvement, 50 had grade 2 improvement, and 14 had grade 1 improvement. Mild post-inflammatory hyperpigmentation was found in only two of the 204 patients, which spontaneously resolved within one month.

\section{Conclusion}

Fractional microneedle RF system was effective and safe in the treatment of facial wrinkles.

\section{Key words}

fractional microneedle radiofrequency; facial wrinkle; periorbital wrinkle; forehead wrinkle; sagging 


\section{INTRODUCTION}

Thermo-lytic treatments with RF devices have been used for the skin rhytides, wrinkles, acne, and acne scars in many dermatological aesthetic clinics. RF devices induce tissue regeneration by neocollagenesis. Electronic currents from RF devices induce bulk heating on the target tissue, known as an electro thermal reaction, depending on the impedance of the tissue. ${ }^{1-5}$ When RF treatment is delivered to the skin, electric currents are absorbed by water, vessels, and collagen fibers. Thereby, electro thermal reactions coagulate dermal tissues and induce cellular aggregation and secretion of cytokines and growth factors for tissue regeneration. ${ }^{6.7}$

Conventional mono polar RF devices deliver RF energy to the skin via a return electrode attached elsewhere on the body, whereas conventional bipolar RF devices comprise a hand piece that is equipped with both the delivery and return electrodes. ${ }^{1-4}$ However, the penetration depth of RF energy has been shown to be limited to less than one-half of the distance between the electrodes in a hand piece of conventional bipolar RF devices. Meanwhile, in both types of conventional RF devices, protection of the epidermis is required in order to safely emit higher levels of RF energy for the proper induction of dermal tissue regeneration.

Fractionated bipolar RF systems deliver RF energy to the skin using microneedles, which allow for precise depth control in the dermis and facilitate safer treatment of lesions; these systems emit higher energy in a noncontiguous pattern to help with epidermal preservation, compared with other fractional lasers. ${ }^{8}$ Fractionated RF systems generate pyramid- or cocoon-shaped sublative zones of electro thermal injury, compared to ablative fractional lasers, which produce conical zones of photo thermal injury that are widest in the epidermis and narrower in the dermis. ${ }^{8,9}$ The procedures involved in administering treatment with RF devices are minimally invasive and necessitate little downtime; they are also highly cost-effective. Since the first fractional bipolar RF delivery system with linear five bipolar microneedle pairs was reported by Hantash et al., several other types of fractionated microneedle RF devices equipped with 25 to 49-microneedles in a disposable tip have been used in the treatment of facial wrinkles, inflammatory acne vulgaris, and atrophic scars. ${ }^{6-10}$

Tissue impedance and permittivity of the skin are associated with skin-RF interactions. ${ }^{711-13}$ Higher impedance and lower permittivity of the lower reticular dermis or subcutaneous fat layer result in a larger but less concentrated zone of thermal injury after RF treatment, compared to the upper papillary dermis, which has lower impedance and higher permittivity. ${ }^{7.11-13}$ Although pre-treatment evaluation of tissue impedance and lesion temperature can be used for optimizing RF parameters, reference data of detailed skin-RF interactions can provide useful information with which to predict therapeutic efficacy and prevent side effects. ${ }^{11}$ In this report, we demonstrate the efficacy and safety of a fractional microneedle RF system in 204 Korean patients with facial wrinkles.

\section{MATERIALS AND METHODS}

Three study centers were selected in Korea. Two hundred and four patients 180 male patients and 124 female patients; a mean age of 47.3 years, ranging from 26 to 68 years; Fitzpatrick skin type: III-IV) who underwent treatment with fractional microneedle RF system IScarlet, Viol, Seongnam, Koreal for facial wrinkles were analyzed in this study (Table 1). Patients were excluded from the study if they had recently received systemic retinoids; 595-nm pulsed dye laser treatment; intense pulsed light, nonablative erbium-glass fractional laser treatment; ablative 10,600-nm carbon dioxide fractional laser treatment; monopolar or bipolar RF treatment; or highintensity focused ultrasound treatment within 6 months. Patients with a high probability of becoming pregnant or a propensity for keloids or immunosuppression were excluded. All patients provided written informed consent regarding their participation in the trial, treatment course, and possible side effects, as well as for the use of their clinical photographs.

Patients were treated with one to three sessions of fractional microneedle RF system at average 4-week intervals. Before application of local anesthesia, the face was cleansed with a mild soap and $70 \%$ alcohol. A topical eutectic mixture of $2.5 \%$ lidocaine hydrochloric acid and $2.5 \%$ prilocaine (AstraZeneca AB, Södertälje, Sweden) was applied to the entire face under occlusion 20-45 min before the therapy. The treatment settings used at each

Table 1. Baseline characteristics of the patients

\begin{tabular}{lccccc}
\hline \multirow{2}{*}{ Center } & \multicolumn{2}{c}{ Gender } & & \multicolumn{2}{c}{ Age (years) } \\
\cline { 2 - 3 } \cline { 5 - 6 } & Male & Female & & Range & Mean \\
\hline Maylin & 25 & 46 & & $31-68$ & 51 \\
Miaero & 37 & 52 & & $29-65$ & 47 \\
Dr. Oh S. & 18 & 26 & & $26-59$ & 42 \\
Total & 80 & 124 & & $26-68$ & 47.3 \\
\hline
\end{tabular}


Table 2. Summarized treatment settings of the fractional microneedle RF system at the three study centers

\begin{tabular}{|c|c|c|c|c|c|c|}
\hline \multirow{2}{*}{ Center } & \multirow{2}{*}{ Pass } & \multicolumn{3}{|c|}{ Treatment Parameter } & \multirow{2}{*}{$\begin{array}{l}\text { Number of } \\
\text { sessions }\end{array}$} & \multirow{2}{*}{$\begin{array}{c}\text { Treatment Interva } \\
\text { (week) }\end{array}$} \\
\hline & & Depth (mm) & Intensity (level) & Duty Time (msec) & & \\
\hline 1 & $1-3$ & $0.8-3.0$ & $6-8$ & $100-200$ & $2-3$ & $3-4$ \\
\hline 2 & $1-3$ & $0.8-3.0$ & $6-8$ & $100-200$ & $2-3$ & $3-4$ \\
\hline 3 & $1-3$ & $0.8-3.0$ & $6-8$ & $100-200$ & $1-3$ & $4-5$ \\
\hline
\end{tabular}

center for skin rejuvenation are summarized in Table 2.

RF therapy was administered over the entire face during a single to double pass, and an additional one to two passes were delivered to moderate or severe lesions with wrinkles. Patients with a history of herpes virus infection were prophylactically prescribed oral valacyclovir hydrochloride (Valtrex, GlaxoSmithKline, Research Triangle Park, NC) for 3 days. The use of a noncomedogenic moisturizer several times a day for a few days after each treatment session was recommended to promote wound healing and prevent dryness. Patients were instructed to avoid overexposure to sunlight and to use a broad-spectrum sunscreen after post-therapy crusting subsided. They were also instructed to avoid the use of any systemic or topical retinoids and antibiotics during the course of treatment.

Photographs were taken under identical camera settings, lighting, and patient positioning at baseline and three months after the last treatment. Two doctors performed objective clinical assessments in a blinded fashion by comparing before-and-after photos using a global improvement scale lgrade 0 , worsened; grade 1 , $0-25 \%=$ minimal improvement or steady state; grade $2,26-50 \%=$ moderate improvement; grade 3, 51-75\% = marked improvement; and grade $4,>75 \%=$ near total improvement). Investigators assessed and recorded possible side effects, including bleeding, oozing, posttherapy dyschromia, scaling, crusting, edema, and erythema at each visit lat 1- to 2-week intervals). Three months after the final treatment, the reported side effects were reassessed and analyzed.

\section{RESULTS}

A total of 204 patients with wrinkles on the face from three centers were treated with one to three sessions of the treatment at average 4-week intervals. Three months after the final treatment, objective clinical assessment using a global improvement scale revealed a median clinical improvement score for the 204 patients of 2.9 \pm 0.9 . Fifty four $(26.5 \%)$ of the 204 patients had grade

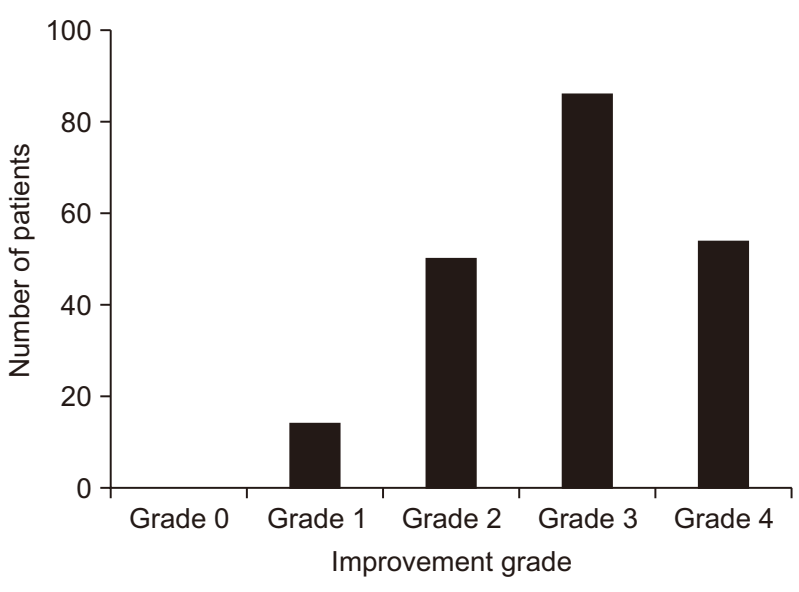

Fig. 1. Clinical improvement grades for the 204 patients treated with fractional microneedle RF system for the rejuvenation treatment of wrinkles along the face.

4 clinical improvement, eighty six (42.2\%) had grade 3 improvement, fifty (24.5\%) had grade 2 improvement, and fourteen (6.9\%) had grade 1 improvement (Fig. 1). Periorbital wrinkles were treated with the settings of a microneedle depth of 1.2-1.5 mm, an intensity level of 7-8, and the duty time of 100-200 msec, and 1-2 passes/session. After fractional microneedle RF system treatment, periorbital wrinkles were improved (Fig. 2). Post-treatment bruising or purpura was not observed. Wrinkles of the forehead were treated with a microneedle depth of 0.8-1.0 mm, an intensity level of 6-7, the duty time of 100-200 msec, and 1-2 passes/session. In this study, most of the patients well tolerated the treatment along the forehead areas and reported good clinical outcomes (Fig. 3). Cheek skin was treated with a microneedle depth of 2.5-3.0 mm, an intensity level of 7-8, the duty time of $200 \mathrm{msec}$, and 2-3 passes/session. After the treatment, rejuvenation effects along the cheeks were observed and sagging jowls were also improved (Fig. 4).

Side effects were examined and reported by participants upon each visit. Although the patients reported some pain, most of the patients acceptably well tolerated the treatment courses by applying topical anesthetic cream before the procedure. Post-treatment 

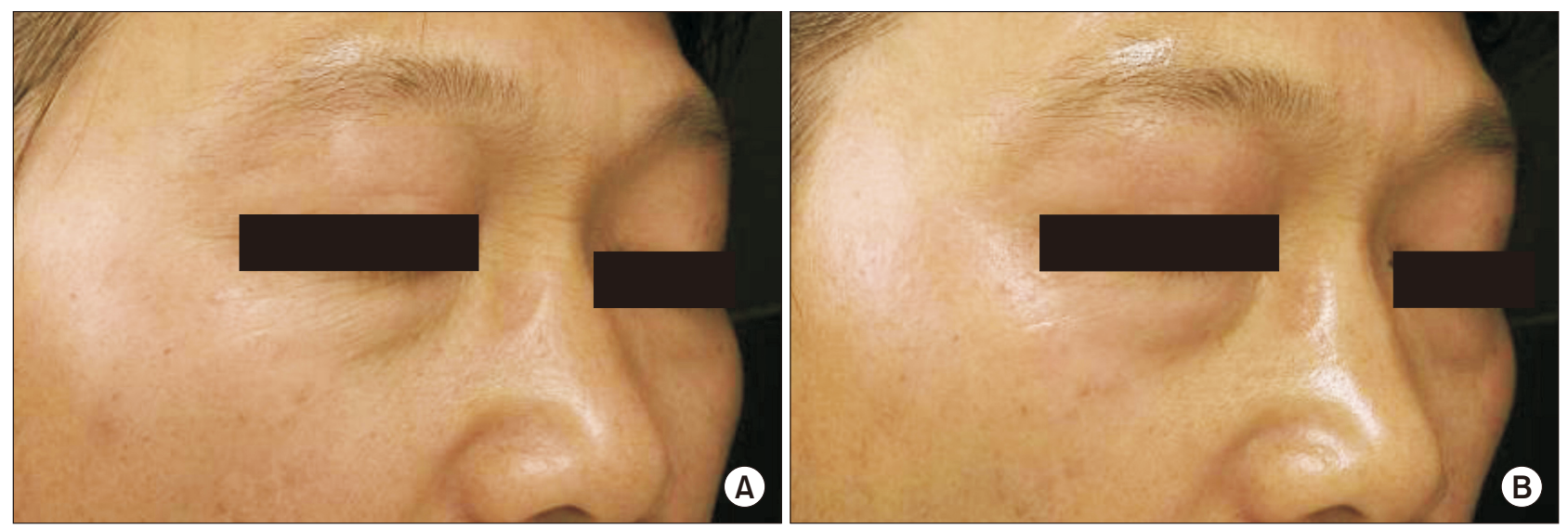

Fig. 2. Marked improvement of periorbital wrinkles in a 48-year-old male patient after two sessions of fractional microneedle RF system treatment. Photos were taken at (A) baseline and (B) two months after treatment.
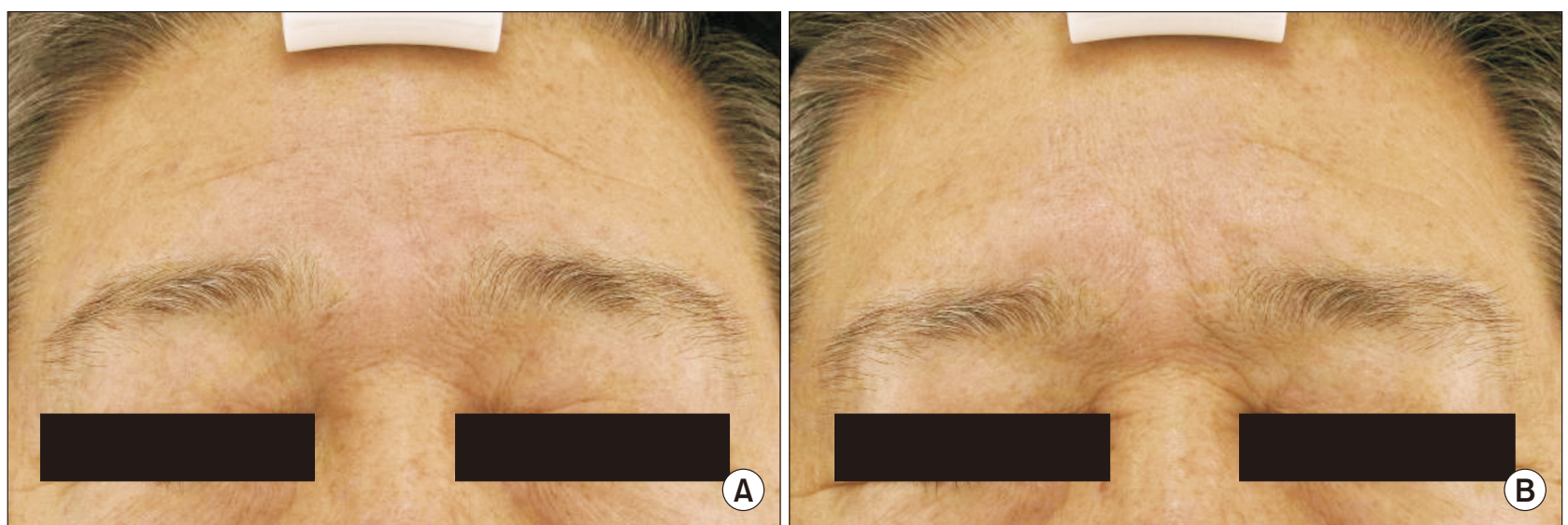

Fig. 3. Remarkable improvement of wrinkles along the forehead in a 56-year-old male patient after three sessions of fractional microneedle RF system treatment. Photos were taken at (A) baseline and (B) three months after treatment.
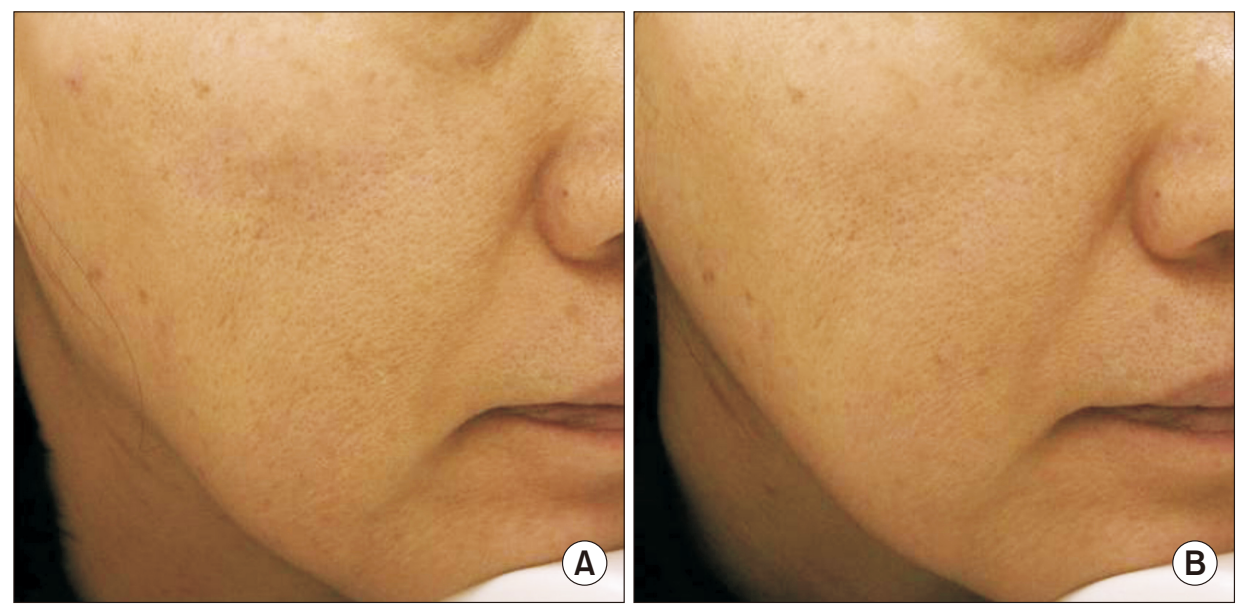

Fig. 4. Improvement of wrinkles and sagging along cheek in a 53-year-old female patient after two sessions of fractional microneedle RF system. Photos were taken at (A) baseline and (B) two months after treatment. 
edema and erythema were mild and transient, usually resolving within 1-3 days. Mild post-inflammatory hyperpigmentation was found in only two (1.0\%) of the 204 patients, which spontaneously resolved within one month. Other possible adverse events, including persistent post-inflammatory hyperpigmentation, scarring, hypopigmentation, secondary bacterial infection, posttherapy blister formation, and viral infection, were not recorded.

\section{DISCUSSION}

The benefit of RF devices is to heat the dermis to a certain temperature and cause immediate collagen stimulation and contraction which induce a neocollagenesis. The mechanism of action of these devices is thought to be related to the fact that water, collagen, melanin, and dermal microvasculature absorb RF energy, producing a bulk heating effect on the dermis and inducing cellular mediator and growth factor secretion, which results in wound healing. ${ }^{6}$ But higher temperatures than 65 degree range denature collagen, even though this brings to more visible results. If the RF energy is placed too deep, there be fat necrosis, and if placed too superficial, the skin can burn or get hyperpigmentation. It is very difficult to heat the dermis to a temperature that is effective and still maintain safe temperatures for the skin surface. Differences in skin thickness, hydration and the composition of collagen and fat can lead to placement of the energy at unknown or variable depths, too.

The concept of treating areas fractionally has been the desired treatment modality for most devices. Almost all of the RF devices have used a bulk heating method and not a fractional heating but recently RF devices by keeping surrounding tissues untreated fractionally show faster healing, less pain, and less risk. The penetration with microneedles to heat skin looks more predictability, more accuracy and more safety.

Hantash and colleagues ${ }^{7}$ first demonstrated the effects of the minimally invasive RF device, a bipolar microneedle electrode system, on human skin. The authors created radiofrequency thermal zones in the dermis using microneedle electrode pairs. ${ }^{7}$ In the present study, a fractional microneedle RF system was used for facial wrinkles. The therapeutic effects of this system may have been the result of tissue heating by the RF energy, as well as from collagen induction by the penetration of microneedles. The microneedle of the RF system used in the present study was not insulated, whereas that of RF device used in the previous report was insulated proximally to protect the epidermis from RF heating at the insertion sites. ${ }^{7}$ Possible side effects associated with RF heating on the epidermis, including burning, noticeable crusting, prolonged erythema, postinflammatory hyperpigmentation, and scarring, were not observed with the use of a RF system with noninsulated microneedles. Instead, therapeutic effects on the epidermis were observed, especially textural improvement. ${ }^{6}$

In conclusion, the present study demonstrated fractional microneedle RF system with noninsulated microneedles was effective and safe in the treatment of facial wrinkles in Fitzpatrick skin types III to IV. The results obtained after the final treatment session continued to improve over time, and patient satisfaction with the procedure was very high with excellent compliance. In addition, as the subcutaneous fat layers of the forehead and periorbital lesion are relatively thin, microneedle RF therapy could easily lead to treatment-associated pain. As cheek skin is thicker than other parts of facial skin, microneedle RF therapy lead to less treatmentassociated pain. The delivery of RF energy to deeper portions of the skin was needed and provided by more pass on the same lesion with fractional microneedle RF system, which delicately controls the penetration depth of microneedles.

\section{REFERENCES}

1. Utley DS, Goode RL. Radiofrequency ablation of the nerve to the corrugator muscle for elimination of glabellar furrowing. Arch Facial Plast Surg 1999;1:46-8.

2. Park YJ, Jo YW, Bang SI, Kim HJ, Lim SY, Mun GH, et al. Radiofrequency volume reduction of gastrocnemius muscle hypertrophy for cosmetic purposes. Aesthetic Plast Surg 2007;31:53-61.

3. Jin Park Y, Woo Jo Y, Bang SI, Kim HJ, Lim SY, Mun GH, et al. Radiofrequency volumetric reduction for masseteric hypertrophy. Aesthetic Plast Surg 2007;31:42-52.

4. Sweet WH, Wepsic JG. Controlled thermocoagulation of trigeminal ganglion and rootlets for differential destruction of pain fibers. 1. Trigeminal neuralgia. J Neurosurg 1974;40:14356.

5. Powell NB, Riley RW, Troell RJ, Blumen MB, Guilleminault C. Radiofrequency volumetric reduction of the tongue. A porcine pilot study for the treatment of obstructive sleep apnea syndrome. Chest 1997;111:1348-55.

6. Lee SJ, Goo JW, Shin J, Chung WS, Kang JM, Kim YK, et al. Use of fractionated microneedle radiofrequency for the treatment of inflammatory acne vulgaris in 18 Korean patients. Dermatol Surg 2012;38:400-5. 
7. Hantash BM, Renton B, Berkowitz RL, Stridde BC, Newman J. Pilot clinical study of a novel minimally invasive bipolar microneedle radiofrequency device. Lasers Surg Med 2009;41:87-95.

8. Brightman L, Goldman MP, Taub AF. Sublative rejuvenation: experience with a new fractional radiofrequency system for skin rejuvenation and repair. J Drugs Dermatol 2009;8111 Suppl):s9-13.

9. Peterson JD, Palm MD, Kiripolsky MG, Guiha IC, Goldman MP. Evaluation of the effect of fractional laser with radiofrequency and fractionated radiofrequency on the improvement of acne scars. Dermatol Surg 2011;37:1260-7.

10. Kim JE, Lee HW, Kim JK, Moon SH, Ko JY, Lee MW, et al. Objective evaluation of the clinical efficacy of fractional radiofrequency treatment for acne scars and enlarged pores in Asian skin. Dermatol Surg 2014:40:988-95.

11. Zheng Z, Goo B, Kim DY, Kang JS, Cho SB. Histometric analysis of skin-radiofrequency interaction using a fractionated microneedle delivery system. Dermatol Surg 2014;40:134-41.

12. Emilia del Pino M, Rosado RH, Azuela A, Graciela Guzmán $M$, Argüelles D, Rodríguez $C$, et al. Effect of controlled volumetric tissue heating with radiofrequency on cellulite and the subcutaneous tissue of the buttocks and thighs. J Drugs Dermatol 2006;5:714-22.

13. Trelles MA, van der Lugt C, Mordon S, Ribé A, Al-Zarouni M. Histological findings in adipocytes when cellulite is treated with a variable-emission radiofrequency system. Lasers Med Sci 2010;25:191-5. 